\title{
Design and Analysis of Broadside Arrays of Uniformly Spaced Linear Elements
}

\author{
Raji A. Abimbola \\ Dept. of Electrical and Electronics Engineering \\ Federal University of Agriculture, Abeokuta, \\ Nigeria
}

\author{
Amusa K. Akinwale \\ Dept. of Electrical and Electronics Engineering \\ Federal University of Agriculture, Abeokuta, \\ Nigeria
}

\begin{abstract}
In this paper, equation for computing the radiated far field of broadside arrays of uniformly spaced elements, assuming sinusoidal current distribution is derived. Computational data are obtained for the antenna parameters such as array factor patterns, overall radiation patterns and directivity of arrays consisting of $5,9,13$, and 21 elements with values of interelement spacing ranging from $0.25 \lambda$ to $2 \lambda$. Computational results for array factor patterns and overall radiation patterns, represented in graphical formats are consistent with those reported in literature and clearly suggest that array structures with inter-element spacing below $\lambda$ would produce remarkable and desirable beam radiations which may find useful applications for long range transmission. Numerical data for directivity as a function of number of elements for values of spacing considered display feature that is consistent with the expectation, as being characteristic of any antenna type.
\end{abstract}

\section{General Terms}

Antenna Design, Numerical Analysis.

\section{Keywords}

Broadside array, array factor, radiated far field, distant communication, inter-element spacing.

\section{INTRODUCTION}

James Clerk Maxwell in 1864 at University of London began humanity first step towards unifying the theory of electricity and magnetism by representing the relations between these two phenomena through a set of formidable differential equations, otherwise called Maxwell's equations. This important milestone paved the way for various applications such radio, television, radar and mobile phones. Critical to these aforementioned applications is an 'antenna' designed to transmit or receive electromagnetic waves. Communication between two remote locations has been made possible by the use of this structure. A single dipole antenna is mostly not used for far distant communication because of limitation of smaller coverage occasioned due to smaller gain or directivity of the antenna. Dipoles in array form are the most desired for far distant communication. Antenna arrays could be deployed for myriad number of applications such as radar, sonar, radios and mobile communication systems [1,2,3]. Different forms of array radiations are broadside, end fire and phased arrays, Hansen-Woodyard array. Antennas designed to radiate broadside is usually characterized with direction of radiation being normal to the axis of the array. This is illustrated in Fig. 1 , in which main lobe radiation is normal to array orientation.

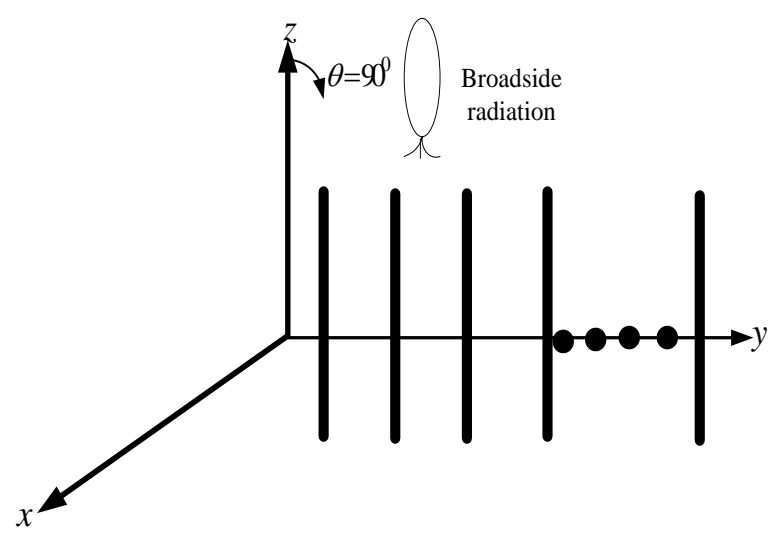

Fig. 1: Illustration of broadside radiation and array orientation

Few of contributions on antenna array are highlighted in this paper. Comprehensive treatment of the usage of antenna array for mobile communication applications was given in [1]. The paper also highlighted some of the advantages such usage could portend. [4] proposed a new technique for designing a concentric circular array antenna of isotropic elements with a view to generating a pencil beam of reduced side lobe and grating lobe levels. Genetic Algorithm was adopted in [5] to find the optimum weights of antenna array of linear elements that could be used to generate radiation patterns of reduced side lobe level.

In this paper, numerical results are obtained for antenna parameters such as, radiation field patterns, and directivity of broadside arrays whose elements are uniformly spaced. These results are represented in graphical formats and depict features which have been reported in the literature as being characteristic of the antenna.

\section{THEORETICAL FORMULATION}

Accurate determination of radiation characteristics of an antenna depends on the nature of current on the structure. Because of difficulties involved in obtaining accurately current distributions by experimental means, frontiers in the field of antenna worked with uniform current, travelling-wave current and standing-wave current distributions [6]. Derivation of radiated field of broadside array assuming sinusoidal current source is explained in what follows.

\subsection{Radiated Field}

The overall radiation patterns of antenna elements in array form can be computed from equation derived by multiplying the radiated field of a single element or a dipole from the reference point by the array factor. This equation is written as,

$$
E_{t o t}=E_{\theta}(\text { at reference point }) \times \text { array factor }
$$


where, $E_{t o t}$ is the total electric field of an array, $E_{\theta}$ is the radiated electric field of a dipole in the $\hat{\theta}$ - direction. The array factor describes the effect of aggregating several radiating elements in an array without taking into cognizance the specific radiation pattern of an element.

For the purpose of investigation, an array of dipole elements is considered here. The radiated field of a half-wave dipole $(l=\lambda / 2)$, lying along $\mathrm{z}$-axis, propagating in free space, is expressible in a form given as

$$
E_{\theta}=j \omega \mu_{0} \sin \theta \int_{-l / 2}^{l / 2} I\left(z^{\prime}\right) \frac{e^{-j k_{0} R}}{4 \pi R} d z^{\prime}
$$

where, $j$ is an imaginary number, $\mu_{0}$ is the free-space permeability, $k_{0}$ represents free space propagation constant, $I\left(z^{\prime}\right)$ is the filamentary source current, and $\mathrm{R}$ is the distance between the source point, represented by $r^{\prime}$ and the field point $r$, and $e^{-j k_{0} R} / R$ is the free space Green function.

The dipole is assumed to be fed with sinusoidal current distribution of the form

$$
I\left(z^{\prime}\right)=I_{0} \cos \left(k_{0} z\right)
$$

Substitution of eqn. (3) in (2) yields,

$$
E_{\theta}=\frac{j \omega \mu_{0}}{4 \pi} \sin \theta \int_{-\lambda / 4}^{\lambda / 4} I_{0} \cos \left(k_{0} z^{\prime}\right) \frac{e^{-j k_{0} R}}{R} d z^{\prime}
$$

where $I_{0}$ is the maximum current, using $\omega \mu_{0}=k_{0} \eta_{0}$ in eqn. (4), we have

$$
E_{\theta}=\frac{j k_{0} \eta_{0} I_{0}}{4 \pi} \sin \theta \int_{-\lambda / 4}^{\lambda / 4} \cos \left(k_{0} z^{\prime}\right) \frac{e^{-j k_{0} R}}{R} d z^{\prime}
$$

$\eta_{0}$ is the free-space intrinsic impedance.

Introducing amplitude and phase approximations associated with far zone electric field as commonly employed in antenna work in eqn. (5), such that

$$
\begin{aligned}
& R=\left|r-r^{\prime}\right| \cong r \text { (amplitude approximation) } \\
& R=\left|r-r^{\prime}\right| \cong r-z^{\prime} \cos \theta \text { (phase approximation) }
\end{aligned}
$$

Eqn. (5) becomes

$E_{\theta}=\frac{j k_{0} \eta_{0} I_{0} e^{-j k_{0} r}}{4 \pi r} \sin \theta \int_{-\lambda / 4}^{\lambda / 4} \cos \left(k_{0} z^{\prime}\right) e^{j k_{0} \cos \theta z^{\prime}} d z^{\prime}$ (7)

The integral expressed by eqn. (7) is easily integrated using the procedure highlighted in eqns. $(8)-(9 b)$ :

$$
\int e^{m z} \cos n z d z=\frac{e^{m z}}{m^{2}+n^{2}}[m \cos n z+n \sin m z]
$$

where

$$
\begin{aligned}
& m=j k_{0} \cos \theta \\
& n=k_{0}
\end{aligned}
$$

Employing eqns. (8) $-(9 \mathrm{~b})$ in (7) results in

$$
\begin{aligned}
E_{\theta}= & \frac{j k_{0} \eta_{0} I_{0} e^{-j k_{0} r} \sin \theta}{4 \pi r}\left[\frac{e^{j k_{0} \cos \theta z^{\prime}}}{\left(j k_{0} \cos \theta\right)^{2}+k_{0}^{2}} \times\right. \\
& \left.\left(j k_{0} \cos \theta \cos k_{0} z^{\prime}+k_{0} \sin k_{0} z^{\prime}\right)\right]_{-\lambda / 4}^{\lambda / 4}
\end{aligned}
$$

Solving eqn. (10) yields

$$
E_{\theta}=\frac{j \eta_{0} I_{0} \cos \left(\frac{\pi}{2} \cos \theta\right)}{2 \pi \sin \theta} \cdot \frac{e^{-j k_{0}}}{r}
$$

The normalized array factor of uniformly spaced array of linear elements lying along $\mathrm{z}$-axis is expressed in a form given as

$$
A F_{n}=\frac{\sin \left(N \frac{\varphi}{2}\right)}{N \sin \left(\frac{\varphi}{2}\right)}
$$

where $A F_{n}$ is the normalized array factor, $N$ is the number of elements in the array, and $\varphi$ is the relative phase between the elements.

The maximum of the normalized array factor as specified in eqn. (12) occurs at $\varphi=0$. That is,

$$
\varphi=k_{0} d \cos \theta+\alpha=0
$$

where, $\alpha$ is the phase angle by which the current in each element leads the current of the preceding element, $d$ is the spacing between the elements, $k_{0}$ has been defined earlier.

For broadside arrays, the maximum radiation is directed towards $\theta=90^{\circ}$, it therefore follows that

$$
\varphi=k_{0} d \cos \theta+\left.\alpha\right|_{\theta=90^{\circ}}=0
$$

With $\alpha=0$, the normalized array factor of eqn. becomes

$$
A F_{n}=\frac{\sin \left(N \frac{k_{0} d \cos \theta}{2}\right)}{N \sin \left(\frac{k_{0} d \cos \theta}{2}\right)}
$$

Employing eqns. (1), (11) and (15), the total radiated field of the array takes the form expressible as,

$$
E_{t}=\frac{j \eta_{0} I_{0} \cos \left(\frac{\pi}{2} \cos \theta\right)}{2 \pi \sin \theta} \cdot \frac{e^{-j k_{0}}}{r} \cdot \frac{\sin \left(N \frac{k_{0} d \cos \theta}{2}\right)}{N \sin \left(\frac{k_{0} d \cos \theta}{2}\right)}
$$

It is important to state here that the normalized value of $E_{\theta}$ would be invoked while utilizing eqn. (16) for the computation of the total radiated field of the broadside array.

\subsection{Directivity}

This is the ratio of maximum radiation intensity in a given direction to radiation intensity averaged all over the direction [7]. Directivity $D$ of an antenna is expressible in a form given as 


$$
D=\frac{U_{\max }}{U_{\text {ave }}}
$$

where $U_{\max }$ is maximum radiation intensity in a given direction, and $U_{\text {ave }}$ is the radiation intensity averaged all over the directions.

Radiation intensity, $U(\theta)$ of broadside array of spacing below $\lambda$ is expressed as [8]

$$
U(\theta)=\left[\frac{\sin \left(N k_{0} d \cos \theta / 2\right)}{N k_{0} d \cos \theta / 2}\right]^{2}
$$

Average radiation intensity, $U_{a v e}$ is of the form

$$
U_{\text {ave }}=\frac{1}{4 \pi} \int_{0}^{\pi} \int_{0}^{2 \pi} U(\theta) \sin \theta d \theta d \varphi
$$

From eqns. (18) and (19), one obtains

$$
U_{\text {ave }}=\frac{1}{4 \pi} \int_{0}^{\pi} \int_{0}^{2 \pi}\left[\frac{\sin \left(N k_{0} d \cos \theta / 2\right)}{N k_{0} d \cos \theta / 2}\right]^{2} \sin \theta d \theta d \varphi
$$

Equation (21) ensues after solving equation (20)

$$
U_{\text {ave }}=\frac{1}{2} \int_{0}^{\pi}\left[\frac{\sin \left(N k_{0} d \cos \theta / 2\right)}{N k_{0} d \cos \theta / 2}\right]^{2} \sin \theta d \theta
$$

Assuming a large array, the solution of eqn. (21) assumes this form

$$
U_{\text {ave }}=\frac{\pi}{N k_{o} d}
$$

At $\theta=90^{\circ}$, the maximum value of the radiation intensity $U_{\max }$ and using eqn. (22) in eqn. (17), directivity of Nelement linear broadside array can be expressed as

$$
D=\frac{N k_{0} d}{\pi}
$$

Substituting for $k_{0}=\frac{2 \pi}{\lambda}$ in eqn. (23), the directivity expressed in terms of the operating wavelength takes this form

$$
D=\frac{2 N d}{\lambda}
$$

In decibel unit, the directivity is written as

$$
D=10 \log 10\left(\frac{2 N d}{\lambda}\right)
$$

\section{NUMERICAL RESULTS}

For the purpose of analysis, computational results are obtained for antenna parameters for broadside arrays of linear elements, whose spacing (d) between the elements ranges from $0.25 \lambda$ to $2 \lambda$. These are discussed in what follows, starting with the array factor patterns.

\subsection{Array factor patterns}

Graphical representations of array factor patterns of broadside arrays consisting of $5,9,13$, and 21 elements, computed by the use of eqn. (15) for $d$ varying from $0.25 \lambda$ to $2 \lambda$ are shown in Figs. 2(a) through 2(e). In each of those illustrations, solid red line, dashed blue line, solid yellow line, and solid black line, respectively, indicate computed results for 5element, 9-element, 13-element, and 21-element broadside arrays. It is evident from the graphical illustrations depicted in Figs. 2(a) to 2(e), that, there are main lobe radiations in the forward and backward directions, this is consistent with the expectation, as array factor takes less cognizance of specific radiation patterns of individual element. This observation is consistent with results reported elsewhere [9]. The results also reveal that the main lobe beamwidth decreases with increasing number of elements and spacing between the elements. This decrease in beamwidth is associated with increase in the number and levels of sidelobes; this appears to be the tradeoff. As evident in Fig. 2(d), there appears to be an occurrence of grating lobes at $d=\lambda$, however, at $d=2 \lambda$, numerous grating lobes are produced as depicted in Fig. 2(e).

\subsection{Total far field patterns of the array}

The far zone fields radiated by the broadside arrays consisting of 5,9,13, and 21 elements computed from eqn. (16) for all values of spacing considered are displayed in Figs. 3 (a) to 3(e). It is of interest to note that, those graphs represent the summation of contribution of far field from each element of the different forms of array considered for analysis. As noted earlier, solid red line, dashed blue line, solid yellow line, and solid black line indicate results for 5-element, 9-element, 13element, and 21-element arrays.

The numerical results graphically depicted in Figs. 3(a) through 3(e), indicate that radiation for all values of $d$ considered is concentrated in the forward direction only, in contrast to results displayed in Figs. 2(a) through 2(e). The results also show that, main lobes of the field patterns become directive with increase in the number of elements and spacing between the elements. This reduction in the main lobe beam width is accompanied with increase in the number and levels of side lobes. There appears to be the occurrence of grating lobes when the spacing between the elements becomes $2 \lambda$.

Because of recurring appearance of grating lobes when $d>\lambda$, it is not out of place if the ensuing section of this paper focuses on the discussion of the radiation lobe called grating lobe.

\subsection{Grating lobes}

A grating lobe is an unwanted side lobe which is a replica of main lobe that beams its radiation in a direction other than the desired direction. In transmitting antenna, side lobe radiation is a waste of electromagnetic energy, and usually constitutes electromagnetic interference, thus causing unintended receivers to pick up stealthy and classified information. In receiving antennas, side lobe allows electromagnetic energy from unwanted directions to enter the system, thus increasing the level of undesirable noise in the receiver. However, there are instances in which grating lobe can be useful, such as in radio interferometry used in radio astronomy [10]. 


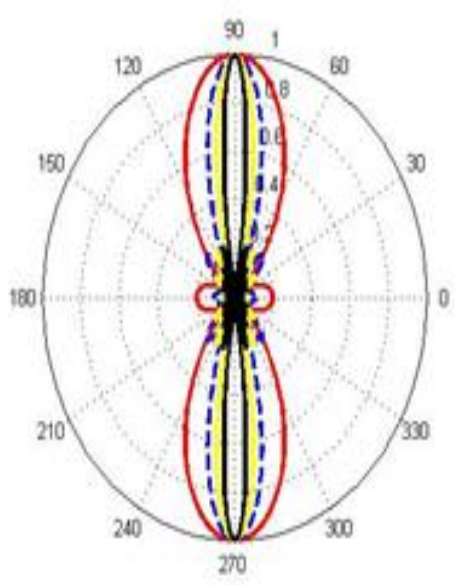

(a)

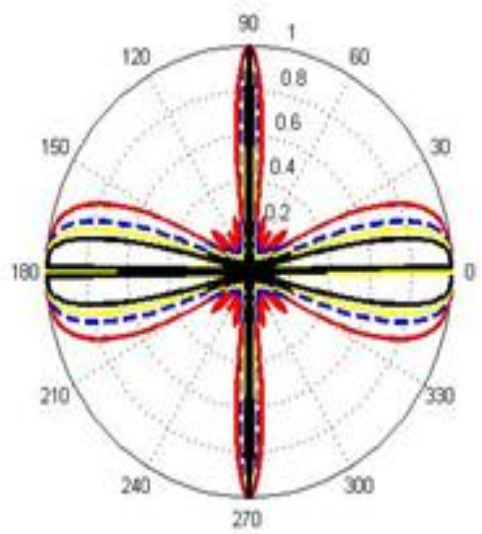

(d)

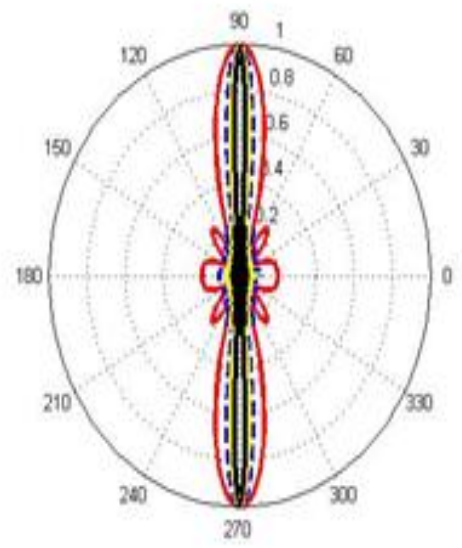

(b)

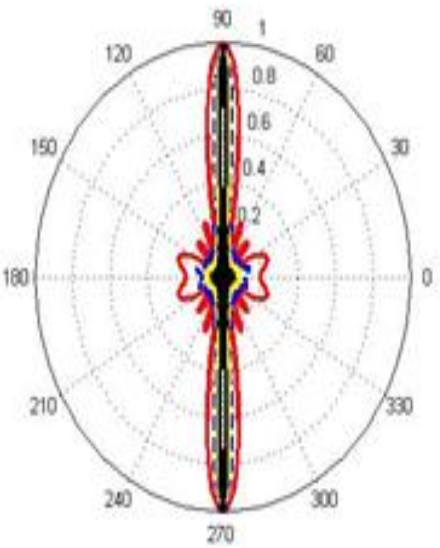

(c)
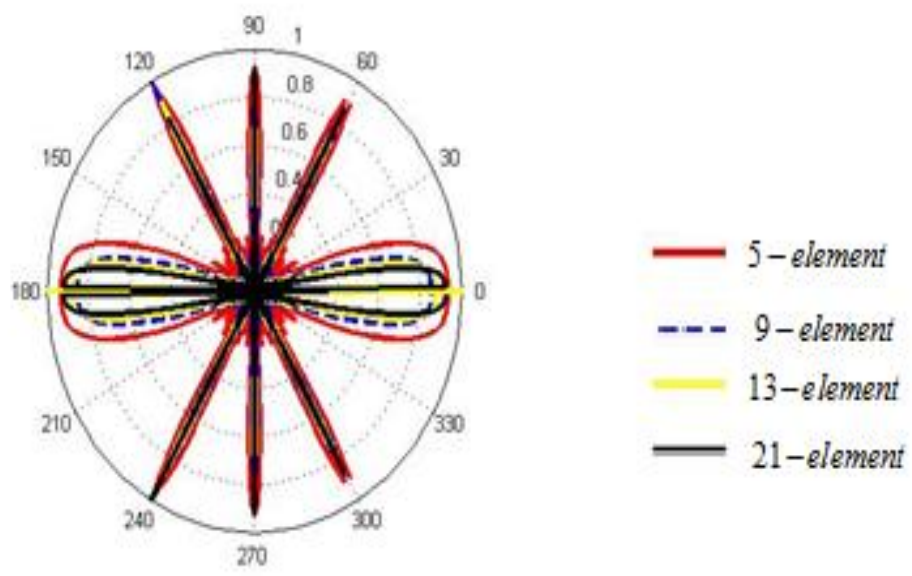

$(e)$

Fig. 2: Array factor patterns of 5, 9, 13, and 21-element broadside arrays at the spacings of: (a) $d=0.25 \lambda$ (b) $d=0.5 \lambda$ (c) $d=0.75 \lambda(\mathbf{d}) d=\lambda(\mathbf{e}) d=2 \lambda$

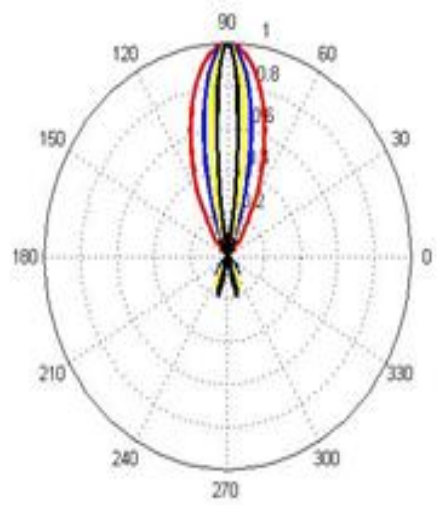

(a)

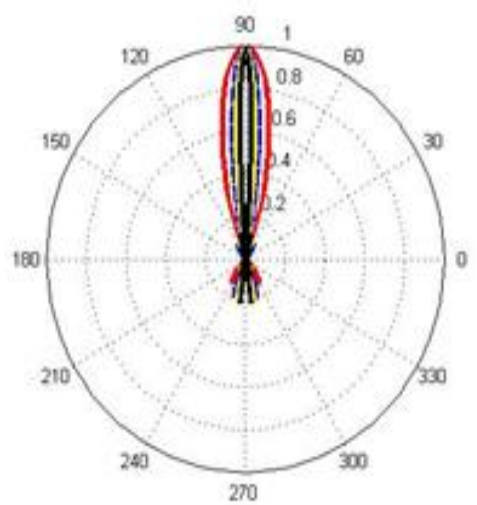

(b)

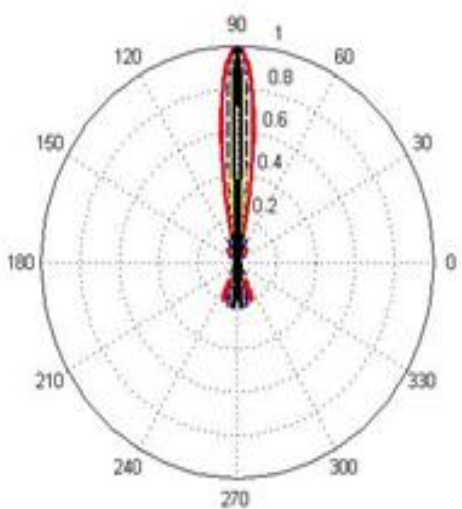

(c) 


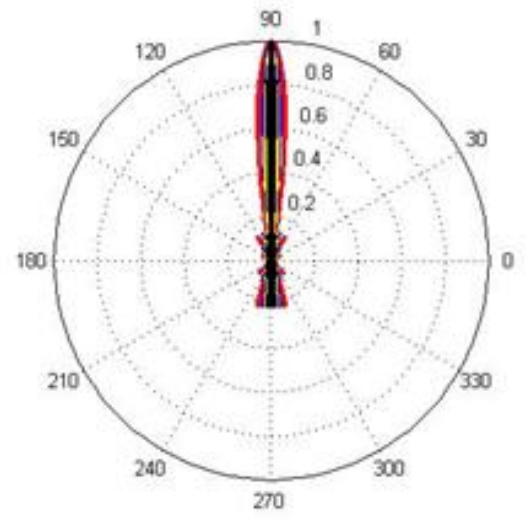

$(d)$

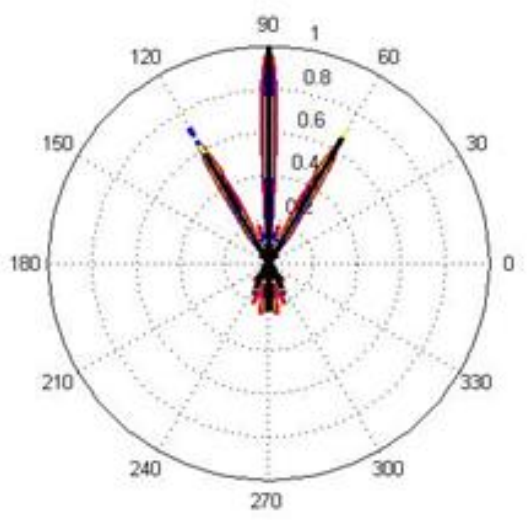

(e)

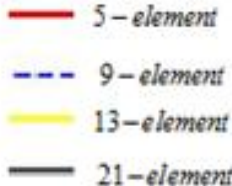

Fig. 3: Radiated far Field patterns of 5, 9, 13, and 21-element broadside arrays at the spacings of: (a) $d=0.25 \lambda$ (b) $d=0.5 \lambda$ (c)

$$
d=0.75 \lambda(\mathbf{d}) d=\lambda(\mathbf{e}) d=2 \lambda
$$

\subsection{Directivity}

Numerical data are computed for directivity using eqn. (25) and these are represented in graphical format as shown in Fig. (4), in which directivity is plotted against number of elements in the arrays. The results show that directivity increases with the number of elements over wide range of values of spacing considered. This cannot be an aberration as previous results depicted in Figs. 2(a) through 2(e) and Figs. 3(a) through 3(e) have shown that main lobe beam width of array patterns reduces as the number of element increases

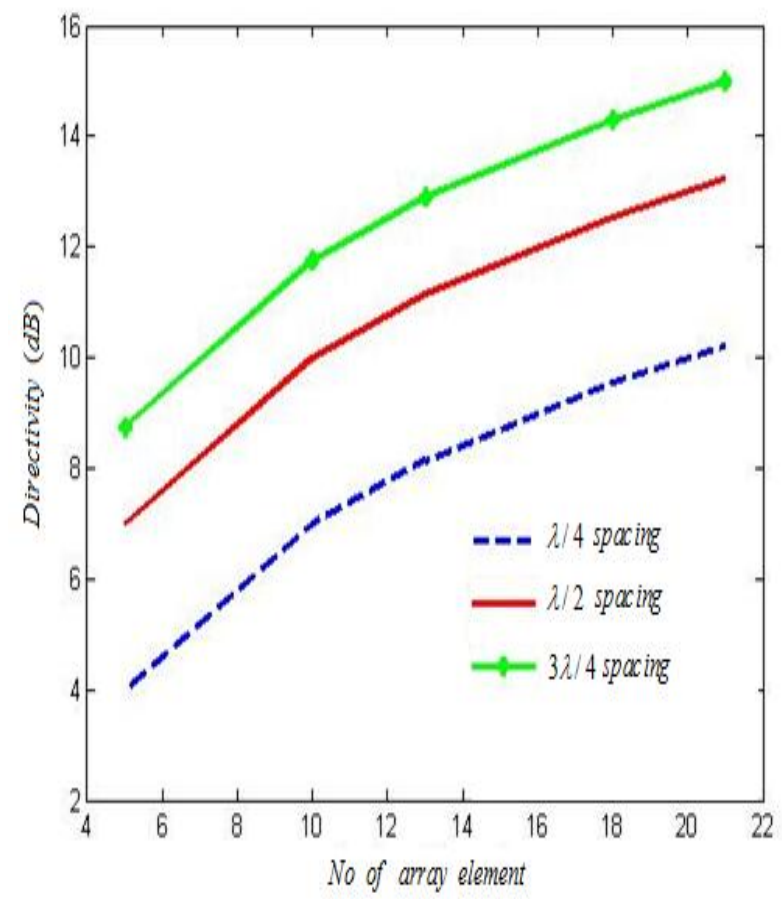

Fig. 4: Variation of the directivity with the number of array element at different inter-element spacing

\section{CONCLUSION AND SUGGESTION FOR FUTURE WORK}

The derivation of the radiated far field of broadside array assuming sinusoidal current source has been presented in this paper. Numerical results are compiled in graphical formats for the array factor pattern, overall far zone radiation patterns and directivity of different forms of array. We find from numerical results displayed for the array factor and radiated far fields for all values of spacing considered that, main lobe beam width reduces as number of elements and spacing between the elements increase. This is consistent with numerical data obtained for directivity which reveals that arrays' directivity increases with number of element. For the sake of comparison, array factor patterns exhibit bidirectional beam radiation (backward and forward radiation), whereas, overall far field patterns depicts radiation in the forward direction. It is of interest to note that when the spacing between the elements is below $\lambda$, both the array factor patterns and overall far field patterns show remarkable and desirable beam radiations, whereas when the spacing is beyond $\lambda$, numerous grating lobes are produced. These results suggest that array structures with spacing below $\lambda$ could find useful application for far distant communication while array structures with spacing beyond $\lambda$ could be desirable in radio interferometry used in radio astronomy. It should be remarked here that, to avoid the occurrence of grating lobes, the spacing between the elements should be less than $\lambda$.

Applications of analysis outlined in the paper as well as the use of other methods in tackling such problems will be explored in the future.

\section{REFERENCES}

[1] Godara L.C. 1997. Applications of antenna arrays to mobile communications Part I: Performance improvement, feasibility, and system considerations. Proceeding of the IEEE, Vol. 85, No 7, 1031-1060.

[2] Tsoulos G.V. 2001. Adaptive antennas for wireless communications, IEEE Press, Piscataway, NJ.

[3] Chandran S. 2004. Adaptive Antenna Arrays: Trends and Applications, Springer.

[4] Krishna P.R., Lakshmi S.S., Sreedevi I., Khan H., Aditya M.P.K., Krishna V.V., Lavanya J. 2012. Sidelobe suppression of concentric circular arrays using nonconventional beamforming technique. International Journal of Modern Engineering Research (IJMER), Vol. 2, No 3, 635-638.

[5] Laseetha T.S.J., Sukanesh R. 2011. Synthesis of linear array using Genetic Algorithm to maximize sidelobe 
reduction. International Journal of Computer Applications, Vol. 20, No. 7, 27-33.

[6] Adekola S.A., Mowete I. A.., Ayorinde A. A. 2007. A rigorous analysis of the radiation characteristics of helical beam antenna. European Journal of Scientific Research, Vol. 16, No 2, 317- 326.

[7] Kraus J.D. 1988, Antennas. McGraw-Hill, Inc., USA, 2nd Edition, 26.
[8] Balanis C.A. 1982. Antenna Theory: Analysis and Design. John Wiley and Sons, New York, 2nd Edition, $276-278$.

[9] Amanpreet K. and Amandeep S. 2012. Analyze the effect of Number of elements on Radiation pattern of Broadside array and End fire array. International Journal of Computer Applications, Vol. 45, No. 18, 31-34

[10] Orfanidis S.J. 2004. Electromagnetic waves and Antennas www.ece.rutgers.edu/ orfanidi/ewa. 